\title{
Construction of Analytic Solution for Time-Fractional Boussinesq Equation Using Iterative Method
}

\author{
Fei Xu, Yixian Gao, and Weipeng Zhang \\ School of Mathematics and Statistics, Center for Mathematics and Interdisciplinary Sciences, Northeast Normal University, \\ Changchun, Jilin 130024, China
}

Correspondence should be addressed to Yixian Gao; gaoyx643@nenu.edu.cn

Received 19 May 2015; Accepted 1 October 2015

Academic Editor: Fawang Liu

Copyright (C) 2015 Fei Xu et al. This is an open access article distributed under the Creative Commons Attribution License, which permits unrestricted use, distribution, and reproduction in any medium, provided the original work is properly cited.

This paper is aimed at constructing analytical solution for both linear and nonlinear time-fractional Boussinesq equations by an iterative method. By the iterative process, we can obtain the analytic solution of the fourth-order time-fractional Boussinesq equation in $\mathbb{R}, \mathbb{R}^{2}$, and $\mathbb{R}^{n}$, the sixth-order time-fractional Boussinesq equation, and the $2 n$ th-order time-fractional Boussinesq equation in $\mathbb{R}$. Through these examples, it shows that the method is simple and effective.

\section{Introduction}

Many phenomena in the physical, chemical, and biological sciences as well as in technologies are governed by differential equations. The idea of derivatives of noninteger order initially appeared in a letter from Leibniz to L'Hospital in 1695 about the notation $d^{n} y / d x^{n}$. L'Hospital posed a question to Leibniz: "what would the result be if $n=1 / 2$ ?" Leibniz replied [1] "it follows that $d^{1 / 2} x$ will be equal to $x \sqrt{d x: x}$. This is an apparent paradox, from which, one day useful consequences will be drawn." In these words, fractional calculus was born. And so most authors on this topic will cite a particular date (September 30,1695 ) as the birthday of so called "fractional calculus" [2]. However, at that time, there are few specific models based on this kind of derivative, so the study of fractional calculus attracts little attention.

Recently, fractional differential equations have been the focus of many studies due to their frequent appearance in various applications in physics, biology, engineering, signal processing, systems identification, control theory, finance, and fractional dynamics, such as chaotic dynamics [3], mechanics of non-Hamiltonian systems [4], anomalous diffusion and transport theory [5], astrophysics [6], physical kinetics [7], plasma physics $[8,9]$, mechanics of fractional media [10], quantum mechanics [11], theory of long range interaction [12].
The Boussinesq equations arise in hydrodynamics to describe propagation of waves in nonlinear and dissipative media $[13,14]$. They are suitable for problems in the percolation of water in porous subsurface strata and widely used in coastal and ocean engineering. Also, Boussinesq equations are the basis of several models used to describe unconfined groundwater flow and subsurface drainage problems. Recently, fractional differential equations have attracted many researchers' interest because of their ability to model particle transport in heterogeneous media and complex phenomena. The fractional Boussinesq equations are suitable for studying the water propagation through heterogeneous porous media. A fractional Boussinesq equation is obtained assuming power law changes of flux in a control volume and using a fractional Taylor series [15]. The fractional differential equations have been solved using several methods such as Laplace transformation method, Fourier transformation method, and operational method [16, 17]. In [18], El-Wakil and Abulwafa used the fractional variational principles and obtained solutions (which are described as periodic, soliton, and explosive waves) of the fractional Boussinesq equation. In [19], based on the finite volume and finite element methods, Zhuang et al. gave two novel numerical methods with a nonlocal operator (using nodal basis functions) for the space-fractional Boussinesq equation. In this paper, we will give a new iterative method to obtain the analytic solution of 
the fourth-, sixth- and $2 n$ th-order fractional Boussinesq equation.

At first, we list some definitions that will be used in the theory of fractional differential equations [2].

Definition 1. The Gamma function is as follows:

$$
\Gamma(x):=\int_{0}^{\infty} t^{x-1} e^{-t} d t, \quad(\operatorname{Re}(x)>0)
$$

Definition 2. The Beta function is as follows:

$$
\begin{aligned}
& B(p, q):=\int_{0}^{1} t^{p-1}(1-t)^{q-1} d t=\frac{\Gamma(p) \Gamma(q)}{\Gamma(p+q)}, \\
&(\operatorname{Re}(p)>0, \operatorname{Re}(q)>0) .
\end{aligned}
$$

Definition 3. The Mittag-Leffler function is as follows:

$$
E_{\alpha}(x):=\sum_{k=0}^{\infty} \frac{x^{k}}{\Gamma(\alpha k+1)}, \quad(\alpha>0)
$$

Definition 4. The Riemann-Liouville fractional integral of order $\alpha(\alpha>0)$ of a function $u(x, t)$ is denoted by $I_{t}^{\alpha} u(x, t)$ and defined as

$$
I_{t}^{\alpha} u(x, t):=\frac{1}{\Gamma(\alpha)} \int_{0}^{t}(t-\tau)^{\alpha-1} u(x, \tau) d \tau, \quad t>0
$$

Definition 5. The Caputo partial fractional derivative of a function $u(x, t)$ is denoted by $D_{t}^{\alpha} u(x, t)$ and defined as

$$
\begin{aligned}
D_{t}^{\alpha} u & (x, t) \\
& := \begin{cases}\frac{\partial^{m}}{\partial t^{m}} u(x, t), & \alpha=m, m \in \mathbb{N} ; \\
I_{t}^{m-\alpha} \frac{\partial^{m}}{\partial t^{m}} u(x, t), & m-1<\alpha<m, m \in \mathbb{N} .\end{cases}
\end{aligned}
$$

Using Definitions 4 and 5, as in [20], we derive

$$
\begin{aligned}
I_{t}^{\alpha} D_{t}^{\alpha} u(x, t)=u(x, t)-\sum_{k=0}^{m-1}\left(\left.\frac{\partial^{k} u(x, t)}{\partial t^{k}}\right|_{t=0}\right) \frac{t^{k}}{k !}, & \\
x & \in \mathbb{R}^{n}, t>0,
\end{aligned}
$$

where $m-1<\alpha \leqslant m, m \in \mathbb{N}$.

Lemma 6. According to Definition 4, one has

$$
I_{t}^{\alpha} t^{\beta}=\frac{\Gamma(\beta+1)}{\Gamma(\alpha+\beta+1)} t^{\alpha+\beta}
$$

Proof. Consider

$$
\begin{aligned}
I_{t}^{\alpha} t^{\beta} & =\frac{1}{\Gamma(\alpha)} \int_{0}^{t}(t-\tau)^{\alpha-1} \tau^{\beta} d \tau \\
& =\frac{1}{\Gamma(\alpha)} \int_{0}^{t}\left(1-\left(\frac{\tau}{t}\right)\right)^{\alpha-1}\left(\frac{\tau}{t}\right)^{(\beta+1)-1} d\left(\frac{\tau}{t}\right) t^{\alpha+\beta} \\
& =\frac{1}{\Gamma(\alpha)} \int_{0}^{1} x^{\alpha-1}(1-x)^{(\beta+1)-1} d x t^{\alpha+\beta} \\
& =\frac{1}{\Gamma(\alpha)} B(\alpha, \beta+1) t^{\alpha+\beta} \\
& =\frac{1}{\Gamma(\alpha)} \frac{\Gamma(\alpha) \Gamma(\beta+1)}{\Gamma(\alpha+\beta+1)} t^{\alpha+\beta}=\frac{\Gamma(\beta+1)}{\Gamma(\alpha+\beta+1)} t^{\alpha+\beta}
\end{aligned}
$$

We will give an iterative method for the general functional differential equation [21]. Using this iterative process, we can construct the solution of the fractional differential equation.

Theorem 7. Consider the functional equation

$$
u(x, t)=f(x, t)+L(u(x, t))+N(u(x, t)),
$$

where $f(x, t)$ is a known function, $(x, t) \in D=\{(x, t): x \in$ $\left.\mathbb{R}^{n}, n \in \mathbb{N}, t \in(0,+\infty)\right\}$, and $L$ and $N$ are linear and nonlinear operators from a Banach space $B$ to itself. When $u(x, t)$ is analytical about the solution of the functional equation (9) can be written into the series form:

$$
u(x, t)=\sum_{n=0}^{\infty} u_{n}(x, t)=u_{0}+u_{1}+u_{2}+\cdots,
$$

where

$$
\begin{aligned}
u_{0} & =f, \\
u_{1} & =L\left(u_{0}\right)+N\left(u_{0}\right), \\
u_{m+1} & =L\left(u_{m}\right)+\left(N\left(\sum_{n=0}^{m} u_{n}\right)-N\left(\sum_{n=0}^{m-1} u_{n}\right)\right), \\
& (m=1,2 \cdots) .
\end{aligned}
$$

If the operators $L$ and $N$ are contractive, then the series $\sum_{n=0}^{\infty} u_{n}(x, t)$ converges absolutely and uniformly.

Proof. Since $u(x, t)=\sum_{n=0}^{\infty} u_{n}(x, t)=u_{0}+u_{1}+u_{2}+\cdots$ and operator $N$ is a nonlinear operator, $N$ can be decomposed as follows:

$$
\begin{aligned}
N(u(x, t))= & N\left(\sum_{n=0}^{\infty} u_{n}(x, t)\right) \\
= & N\left(u_{0}\right) \\
& +\sum_{n=1}^{\infty}\left(N\left(\sum_{j=0}^{n} u_{j}\right)-N\left(\sum_{j=0}^{n-1} u_{j}\right)\right) .
\end{aligned}
$$


For the linear operator $L$, it can also be written into the form

$$
\begin{aligned}
L(u(x, t))= & L\left(\sum_{n=0}^{\infty} u_{n}(x, t)\right) \\
= & L\left(u_{0}\right) \\
& +\sum_{n=1}^{\infty}\left(N\left(\sum_{j=0}^{n} u_{j}\right)-N\left(\sum_{j=0}^{n-1} u_{j}\right)\right) .
\end{aligned}
$$

Set the operator

$$
M(u(x, t)):=L(u(x, t))+N(u(x, t)) .
$$

According to (12), (13), and (14), we have

$$
\begin{aligned}
M(u)= & M\left(u_{0}\right) \\
& +\sum_{n=1}^{\infty}\left(M\left(\sum_{j=0}^{n} u_{j}\right)-M\left(\sum_{j=0}^{n-1} u_{j}\right)\right) .
\end{aligned}
$$

According to (11), (14), and (15), we can get

$$
\begin{aligned}
u_{0} & =f \\
u_{1} & =M\left(u_{0}\right), \\
u_{m+1} & =M\left(\sum_{n=0}^{m} u_{n}\right)-M\left(\sum_{n=0}^{m-1} u_{n}\right), \quad(m=1,2 \cdots) .
\end{aligned}
$$

Since operators $L$ and $N$ are contractive, $M$ is also contractive; that is, there exists a constant $0<K<1$, such that

$$
\left\|M\left(v_{i}\right)-M\left(v_{j}\right)\right\| \leq K\left\|v_{i}-v_{j}\right\|, \quad \forall v_{i}, v_{j} \in B,
$$

where $\|\cdot\|$ denotes the norm on Banach space $B$. Finally, let us give the estimation of $u_{m+1}$ :

$$
\begin{aligned}
\left\|u_{m+1}\right\| & =\left\|M\left(\sum_{n=0}^{m} u_{n}\right)-M\left(\sum_{n=0}^{m-1} u_{n}\right)\right\| \leq K\left\|u_{m}\right\| \\
& \leq K^{m+1}\left\|u_{0}\right\| .
\end{aligned}
$$

Since $0<K<1$, the series $\sum_{m=1}^{\infty} K^{m+1}\left\|u_{0}\right\|$ converges absolutely as well as uniformly.

According to Weierstrass' criterion, we show that the series $\sum_{i=0}^{\infty} u_{i}$ converges absolutely as well as uniformly.

In fact, using (11) along with Theorem 7, a new iterative method for constructing analytical solutions of the functional equation (12) is given:

$$
u(x, t)=\sum_{n=0}^{\infty} u_{n}=u_{0}+u_{1}+u_{2}+\cdots
$$

where

$$
\begin{aligned}
& u_{0}=f, \\
& u_{1}=L\left(u_{0}\right)+N\left(u_{0}\right), \\
& u_{2}=L\left(u_{1}\right)+\left(N\left(u_{0}+u_{1}\right)-N\left(u_{0}\right)\right), \\
& u_{3}=L\left(u_{2}\right)+\left(N\left(u_{0}+u_{1}+u_{2}\right)-N\left(u_{0}+u_{1}\right)\right), \\
& \vdots, \\
& u_{m+1}=L\left(u_{m}\right)+\left(N\left(u_{0}+u_{1}+u_{2}+\cdots+u_{m}\right)\right. \\
& \left.\quad-N\left(u_{0}+u_{1}+\cdots+u_{m-1}\right)\right),
\end{aligned}
$$

$\vdots$

According to Theorem 7, we know that the iterative process is reasonable. In the following, we apply the iterative method to the linear and nonlinear time-fractional Boussinesq equation.

\section{Application to the Time-Fractional Boussinesq Equations}

In this section, using the iterative method, we will construct the solution of the time-fractional Boussinesq equation.

2.1. Fourth-Order Time-Fractional Boussinesq Equation in $\mathbb{R}$. Consider the general fourth-order time-fractional Boussinesq equation with one-dimensional space variable

$$
\begin{aligned}
D_{t}^{(\alpha)} u(x, t)= & \beta D_{x}^{(4)} u(x, t)+\gamma D_{x}^{(2)} u(x, t) \\
& +\theta D_{x}^{(2)} u^{2}(x, t)-4 \theta u^{2}(x, t),
\end{aligned}
$$

with the initial conditions

$$
\begin{gathered}
u(x, 0)=e^{x}, \\
u_{t}(x, 0)=0,
\end{gathered}
$$

$$
x \in \mathbb{R}, t>0,
$$

where $1<\alpha \leq 2$ describing the order of the fractional time derivative, $u(x, t)$ is a field function, and $\beta, \gamma$, and $\theta$ are constant coefficients.

Applying $I_{t}^{\alpha}$ to both sides of (21), we derive

$$
\begin{aligned}
I_{t}^{\alpha} D_{t}^{\alpha} u(x, t)= & \beta I_{t}^{\alpha} D_{x}^{(4)} u(x, t)+\gamma I_{t}^{\alpha} D_{x}^{(2)} u(x, t) \\
& +\theta I_{t}^{\alpha} D_{x}^{(2)} u^{2}(x, t)-4 \theta I_{t}^{\alpha} u^{2}(x, t)
\end{aligned}
$$

Denoting

$$
\begin{aligned}
& L(u(x, t))=\beta I_{t}^{\alpha} D_{x}^{(4)} u(x, t)+\gamma I_{t}^{\alpha} D_{x}^{(2)} u(x, t), \\
& N(u(x, t))=\theta I_{t}^{\alpha} D_{x}^{(2)} u^{2}(x, t)-4 \theta I_{t}^{\alpha} u^{2}(x, t)
\end{aligned}
$$

and using (9), we can get

$$
I_{t}^{\alpha} D_{t}^{\alpha} u(x, t)=L(u(x, t))+N(u(x, t)) .
$$


According to the iterative method (19)-(20), we obtain

$$
\begin{aligned}
& u_{0}=e^{x} \\
& u_{1}=e^{x} \frac{(\beta+\gamma) t^{\alpha}}{\Gamma(\alpha+1)}, \\
& u_{2}=e^{x} \frac{\left((\beta+\gamma) t^{\alpha}\right)^{2}}{\Gamma(2 \alpha+1)}, \ldots
\end{aligned}
$$

Generally,

$$
u_{m}=e^{x} \frac{\left((\beta+\gamma) t^{\alpha}\right)^{m}}{\Gamma(m \alpha+1)}
$$

So the solution of $(21)$ is

$$
\begin{aligned}
u(x, t) & =\sum_{n=0}^{\infty} u_{n}(x, t)=\sum_{n=0}^{\infty} e^{x} \frac{\left((\beta+\gamma) t^{\alpha}\right)^{n}}{\Gamma(n \alpha+1)} \\
& =e^{x} E_{\alpha}\left((\beta+\gamma) t^{\alpha}\right) .
\end{aligned}
$$

Remark 8. Particularly, if $\alpha=2$, then the fourth-order timefractional Boussinesq equation is the regular Boussinesq equation. The solution is $u(x, t)=e^{x} E_{2}\left((\beta+\gamma) t^{2}\right)$, which is consistent with the solutions in [22].

Remark 9. If $\beta=0, \gamma \neq 0$, then the fourth-order timefractional Boussinesq equation becomes fractional wave equation, and the solution is $u(x, t)=e^{x} E_{\alpha}\left(\gamma t^{\alpha}\right)$.

Remark 10. If $\theta=0$, then the fourth-order time-fractional Boussinesq equation becomes fractional linear beam equation, and the solution is $u(x, t)=e^{x} E_{\alpha}\left((\beta+\gamma) t^{\alpha}\right)$.

2.2. Fourth-Order Time-Fractional Boussinesq Equation in $\mathbb{R}^{2}$. Consider the fourth-order time-fractional Boussinesq equation with two-dimensional space variables

$$
\begin{aligned}
D_{t}^{(\alpha)} u(\mathbf{x}, t)= & \beta_{1} D_{x_{1}}^{(4)} u(\mathbf{x}, t)+\beta_{2} D_{x_{2}}^{(4)} u(\mathbf{x}, t) \\
& +\gamma_{1} D_{x_{1}}^{(2)} u(\mathbf{x}, t)+\gamma_{2} D_{x_{2}}^{(2)} u(\mathbf{x}, t) \\
& +\theta_{1} D_{x_{1}}^{(2)} u^{2}(\mathbf{x}, t)+\theta_{2} D_{x_{2}}^{(2)} u^{2}(\mathbf{x}, t) \\
& -4 \theta_{1} u^{2}(\mathbf{x}, t)-4 \theta_{2} u^{2}(\mathbf{x}, t), \\
& \mathbf{x}=\left(x_{1}, x_{2}\right) \in \mathbb{R}^{2}, t>0,
\end{aligned}
$$

with the initial conditions

$$
\begin{aligned}
& u\left(x_{1}, x_{2}, 0\right)=e^{x_{1}+x_{2}}, \\
& u_{t}\left(x_{1}, x_{2}, 0\right)=0,
\end{aligned}
$$

where $1<\alpha \leq 2$ and the coefficients $\beta_{i}, \gamma_{i}$, and $\theta_{i} \in \mathbb{R}$, $(i=1,2)$.
Applying $I_{t}^{\alpha}$ to both sides of (29), we derive

$$
\begin{aligned}
I_{t}^{\alpha} D_{t}^{\alpha} u(\mathbf{x}, t)= & \beta_{1} I_{t}^{\alpha} D_{x_{1}}^{(4)} u(\mathbf{x}, t)+\beta_{2} I_{t}^{\alpha} D_{x_{2}}^{(4)} u(\mathbf{x}, t) \\
& +\gamma_{1} I_{t}^{\alpha} D_{x_{1}}^{(2)} u(\mathbf{x}, t)+\gamma_{2} I_{t}^{\alpha} D_{x_{2}}^{(2)} u(\mathbf{x}, t) \\
& +\theta_{1} I_{t}^{\alpha} D_{x_{1}}^{(2)} u^{2}(\mathbf{x}, t)+\theta_{2} I_{t}^{\alpha} D_{x_{2}}^{(2)} u^{2}(\mathbf{x}, t) \\
& -4 \theta_{1} I_{t}^{\alpha} u^{2}(\mathbf{x}, t)-4 \theta_{2} I_{t}^{\alpha} u^{2}(\mathbf{x}, t) .
\end{aligned}
$$

Now, let us denote that

$$
\begin{aligned}
L(u(\mathbf{x}, t)):= & \beta_{1} I_{t}^{\alpha} D_{x_{1}}^{(4)} u(\mathbf{x}, t)+\beta_{2} I_{t}^{\alpha} D_{x_{2}}^{(4)} u(\mathbf{x}, t) \\
& +\gamma_{1} I_{t}^{\alpha} D_{x_{1}}^{(2)} u(\mathbf{x}, t) \\
& +\gamma_{2} I_{t}^{\alpha} D_{x_{2}}^{(2)} u(\mathbf{x}, t), \\
N(u(\mathbf{x}, t)):= & \theta_{1} I_{t}^{\alpha} D_{x_{1}}^{(2)} u^{2}(\mathbf{x}, t)+\theta_{2} I_{t}^{\alpha} D_{x_{2}}^{(2)} u^{2}(\mathbf{x}, t) \\
& -4 \theta_{1} I_{t}^{\alpha} u^{2}(\mathbf{x}, t)-4 \theta_{2} I_{t}^{\alpha} u^{2}(\mathbf{x}, t) .
\end{aligned}
$$

So we can get

$$
I_{t}^{\alpha} D_{t}^{\alpha} u(\mathbf{x}, t)=L(u(\mathbf{x}, t))+N(u(\mathbf{x}, t)) .
$$

According to the iterative method (19)-(20), we can get

$$
\begin{aligned}
& u_{0}=e^{x_{1}+x_{2}}, \\
& u_{1}=e^{x_{1}+x_{2}} \frac{\left(\beta_{1}+\beta_{2}+\gamma_{1}+\gamma_{2}\right) t^{\alpha}}{\Gamma(\alpha+1)}, \\
& u_{2}=e^{x_{1}+x_{2}} \frac{\left(\left(\beta_{1}+\beta_{2}+\gamma_{1}+\gamma_{2}\right) t^{\alpha}\right)^{2}}{\Gamma(2 \alpha+1)}, \ldots
\end{aligned}
$$

Generally,

$$
u_{m}=e^{x_{1}+x_{2}} \frac{\left(\left(\beta_{1}+\beta_{2}+\gamma_{1}+\gamma_{2}\right) t^{\alpha}\right)^{m}}{\Gamma(m \alpha+1)} .
$$

So the solution of (29) is

$$
\begin{aligned}
u(\mathbf{x}, t) & =\sum_{n=0}^{\infty} u_{n}(\mathbf{x}, t) \\
& =\sum_{n=0}^{\infty} e^{x_{1}+x_{2}} \frac{\left(\left(\beta_{1}+\beta_{2}+\gamma_{1}+\gamma_{2}\right) t^{\alpha}\right)^{n}}{\Gamma(n \alpha+1)} \\
& =e^{x_{1}+x_{2}} E_{\alpha}\left(\left(\beta_{1}+\beta_{2}+\gamma_{1}+\gamma_{2}\right) t^{\alpha}\right) .
\end{aligned}
$$

2.3. Fourth-Order Time-Fractional Boussinesq Equation in $\mathbb{R}^{n}$. Consider the fourth-order time-fractional Boussinesq equation with $n$-dimensional space variables

$$
\begin{aligned}
D_{t}^{(\alpha)} u(\mathbf{x}, t)= & \sum_{i=0}^{n} \beta_{i} D_{x_{i}}^{(4)} u(\mathbf{x}, t)+\sum_{i=0}^{n} \gamma_{i} D_{x_{i}}^{(2)} u(\mathbf{x}, t) \\
& +\sum_{i=0}^{n} \theta_{i} D_{x_{i}}^{(2)} u^{2}(\mathbf{x}, t)-\sum_{i=0}^{n} 4 \theta_{i} u^{2}(\mathbf{x}, t),
\end{aligned}
$$


with the initial conditions

$$
\begin{aligned}
& u(\mathbf{x}, 0)=\exp \left(\sum_{i=0}^{n} x_{i}\right), \\
& u_{t}(\mathbf{x}, 0)=0,
\end{aligned}
$$

where $1<\alpha \leq 2, \beta_{i}, \gamma_{i}, \theta_{i} \in \mathbb{R},(i=1,2, \ldots, n)$, and $\mathbf{x}=$ $\left(x_{1}, x_{2}, \ldots, x_{n}\right) \in \mathbb{R}^{n}, t>0$.

Applying $I_{t}^{\alpha}$ to both sides of (37), we have

$$
\begin{aligned}
I_{t}^{\alpha} D_{t}^{\alpha} u(\mathbf{x}, t)= & \sum_{i=0}^{n} \beta_{i} I_{t}^{\alpha} D_{x_{i}}^{(4)} u(\mathbf{x}, t)+\sum_{i=0}^{n} \gamma_{i} I_{t}^{\alpha} D_{x_{i}}^{(2)} u(\mathbf{x}, t) \\
& +\sum_{i=0}^{n} \theta_{i} I_{t}^{\alpha} D_{x_{i}}^{(2)} u^{2}(\mathbf{x}, t) \\
& -4 \sum_{i=0}^{n} \theta_{i} I_{t}^{\alpha} u^{2}(\mathbf{x}, t)
\end{aligned}
$$

Denoting

$$
\begin{aligned}
L(u(\mathbf{x}, t)) & =\sum_{i=0}^{n} \beta_{i} I_{t}^{\alpha} D_{x_{i}}^{(4)} u(\mathbf{x}, t)+\sum_{i=0}^{n} \gamma_{i} I_{t}^{\alpha} D_{x_{i}}^{(2)} u(x, t), \\
N(u(\mathbf{x}, t)) & =\sum_{i=0}^{n} \theta_{i} I_{t}^{\alpha} D_{x_{i}}^{(2)} u^{2}(\mathbf{x}, t)-4 \sum_{i=0}^{n} \theta_{i} I_{t}^{\alpha} u^{2}(\mathbf{x}, t),
\end{aligned}
$$

we can get

$$
I_{t}^{\alpha} D_{t}^{\alpha} u(\mathbf{x}, t)=L(u(\mathbf{x}, t))+N(u(\mathbf{x}, t)) .
$$

According to the iterative method (19)-(20), we obtain

$$
\begin{aligned}
& u_{0}=\exp \left(\sum_{i=0}^{n} x_{i}\right) \\
& u_{1}=\exp \left(\sum_{i=0}^{n} x_{i}\right) \frac{\left(\sum_{i=0}^{n} \beta_{i}+\sum_{i=0}^{n} \gamma_{i}\right) t^{\alpha}}{\Gamma(\alpha+1)}, \\
& u_{2}=\exp \left(\sum_{i=0}^{n} x_{i}\right) \frac{\left(\left(\sum_{i=0}^{n} \beta_{i}+\sum_{i=0}^{n} \gamma_{i}\right) t^{\alpha}\right)^{2}}{\Gamma(2 \alpha+1)}, \ldots
\end{aligned}
$$

Generally,

$$
u_{m}=\exp \left(\sum_{i=0}^{n} x_{i}\right) \frac{\left(\left(\sum_{i=0}^{n} \beta_{i}+\sum_{i=0}^{n} \gamma_{i}\right) t^{\alpha}\right)^{m}}{\Gamma(m \alpha+1)}
$$

So the solution of (37) is

$$
\begin{aligned}
u(\mathbf{x}, t) & =\sum_{n=0}^{\infty} u_{n}(\mathbf{x}, t) \\
& =\sum_{n=0}^{\infty} \exp \left(\sum_{i=0}^{n} x_{i}\right) \frac{\left(\left(\sum_{i=0}^{n} \beta_{i}+\sum_{i=0}^{n} \gamma_{i}\right) t^{\alpha}\right)^{m}}{\Gamma(m \alpha+1)} \\
& =\exp \left(\sum_{i=0}^{n} x_{i}\right) E_{\alpha}\left(\left(\sum_{i=0}^{n} \beta_{i}+\sum_{i=0}^{n} \gamma_{i}\right) t^{\alpha}\right) .
\end{aligned}
$$

2.4. Sixth-Order Time-Fractional Boussinesq Equation in $\mathbb{R}$. Let us consider the sixth-order time-fractional Boussinesq equation

$$
\begin{aligned}
D_{t}^{(\alpha)} u(x, t)= & D_{x}^{(6)} u(x, t)+D_{x}^{(4)} u(x, t)+D_{x}^{(2)} u(x, t) \\
& +\theta D_{x}^{(2)} u^{2}(x, t)-4 \theta u^{2}(x, t)
\end{aligned}
$$

where $x \in \mathbb{R}, t>0,1<\alpha \leq 2$, and the parameter $\theta \in \mathbb{R}$, with the initial conditions

$$
\begin{gathered}
u(x, 0)=e^{x}, \\
u_{t}(x, 0)=0 .
\end{gathered}
$$

Applying $I_{t}^{\alpha}$ to both sides of (45), we have

$$
\begin{aligned}
I_{t}^{\alpha} D_{t}^{\alpha} u(x, t)= & I_{t}^{\alpha} D_{x}^{(6)} u(x, t)+I_{t}^{\alpha} D_{x}^{(4)} u(x, t) \\
& +I_{t}^{\alpha} D_{x}^{(2)} u(x, t)+\theta I_{t}^{\alpha} D_{x}^{(2)} u^{2}(x, t) \\
& -I_{t}^{\alpha} 4 \theta u^{2}(x, t) .
\end{aligned}
$$

Denote that

$$
\begin{aligned}
L(u(x, t))= & I_{t}^{\alpha} D_{x}^{(6)} u(x, t)+I_{t}^{\alpha} D_{x}^{(4)} u(x, t) \\
& +\theta I_{t}^{\alpha} D_{x}^{(2)} u(x, t), \\
N(u(x, t))= & \theta I_{t}^{\alpha} D_{x}^{(2)} u^{2}(x, t)-I_{t}^{\alpha} 4 \theta u^{2}(x, t) .
\end{aligned}
$$

So we can get

$$
I_{t}^{\alpha} D_{t}^{\alpha} u(x, t)=L(u(x, t))+N(u(x, t)) .
$$

According to the iterative method (19)-(20), we have

$$
\begin{aligned}
& u_{0}=e^{x} \\
& u_{1}=e^{x} \frac{3 t^{\alpha}}{\Gamma(\alpha+1)}, \\
& u_{2}=e^{x} \frac{\left(3 t^{\alpha}\right)^{2}}{\Gamma(2 \alpha+1)}, \ldots
\end{aligned}
$$

Generally,

$$
u_{m}=e^{x} \frac{\left(3 t^{\alpha}\right)^{m}}{\Gamma(m \alpha+1)} .
$$

So, the solution of (45) is

$$
\begin{aligned}
u(x, t) & =\sum_{n=0}^{\infty} u_{n}(x, t)=\sum_{n=0}^{\infty} e^{x} \frac{\left(3 t^{\alpha}\right)^{n}}{\Gamma(n \alpha+1)} \\
& =e^{x} E_{\alpha}\left(3 t^{\alpha}\right) .
\end{aligned}
$$

Remark 11. If $\alpha=2$, the sixth-order time-fractional Boussinesq equation is the regular Boussinesq equation, and the solution is $u(x, t)=e^{x} E_{2}\left(3 t^{2}\right)$. 
2.5. 2nth-Order Time-Fractional Boussinesq Equation in $\mathbb{R}$. Consider the $2 n$ th-order time-fractional Boussinesq equation

$$
\begin{aligned}
D_{t}^{(\alpha)} u(x, t)= & D_{t}^{(2 n)} u(x, t)+D_{t}^{(2 n-2)} u(x, t)+\cdots \\
& +D_{t}^{(2)} u(x, t)+\theta D_{x}^{(2)} u^{2}(x, t) \\
& -4 \theta u^{2}(x, t),
\end{aligned}
$$

where $1<\alpha \leq 2, t>0$, and $x \in \mathbb{R}$, with the initial conditions

$$
\begin{gathered}
u(x, 0)=e^{x}, \\
u_{t}(x, 0)=0 .
\end{gathered}
$$

And we apply $I_{t}^{\alpha}$ to both sides of (53):

$$
\begin{aligned}
I_{t}^{\alpha} D_{t}^{\alpha} u(x, t)= & I_{t}^{\alpha} D_{x}^{(2 n)} u(x, t)+\cdots+I_{t}^{\alpha} D_{x}^{(2)} u(x, t) \\
& +\theta I_{t}^{\alpha} D_{x}^{(2)} u^{2}(x, t)-I_{t}^{\alpha} 4 \theta u^{2}(x, t) .
\end{aligned}
$$

Denoting

$$
\begin{aligned}
L(u(x, t))= & I_{t}^{\alpha} D_{x}^{(2 n)} u(x, t)+I_{t}^{\alpha} D_{x}^{(2 n-2)} u(x, t)+\cdots \\
& +I_{t}^{\alpha} D_{x}^{(2)} u(x, t), \\
N(u(x, t))= & \theta I_{t}^{\alpha} D_{x}^{(2)} u^{2}(x, t)-I_{t}^{\alpha} 4 \theta u^{2}(x, t),
\end{aligned}
$$

we can get

$$
I_{t}^{\alpha} D_{t}^{\alpha} u(x, t)=L(u(x, t))+N(u(x, t)) .
$$

According to iterative method (19)-(20), we derive

$$
\begin{aligned}
& u_{0}=e^{x} \\
& u_{1}=e^{x} \frac{n t^{\alpha}}{\Gamma(\alpha+1)}, \\
& u_{2}=e^{x} \frac{\left(n t^{\alpha}\right)^{2}}{\Gamma(2 \alpha+1)}, \ldots
\end{aligned}
$$

Generally,

$$
u_{m}=e^{x} \frac{\left(n t^{\alpha}\right)^{m}}{\Gamma(m \alpha+1)} .
$$

So, the solution of (53) is

$$
u(x, t)=\sum_{i=0}^{\infty} u_{i}(x, t)=\sum_{i=0}^{\infty} e^{x} \frac{\left(n t^{\alpha}\right)^{i}}{\Gamma(i \alpha+1)}=e^{x} E_{\alpha}\left(n t^{\alpha}\right) .
$$

Remark 12. If $\alpha=2$, the $2 n$ th-order time-fractional Boussinesq equation is the regular Boussinesq equation, and the solution is $u(x, t)=e^{x} E_{2}\left(n t^{2}\right)$.

\section{Conclusion}

For a given order time-fractional Boussinesq equation with the initial value $u(x, 0)=e^{x}, u_{t}(x, 0)=0$, such as the fourth-order time-fractional Boussinesq equation, the sixthorder time-fractional Boussinesq equation, and the $2 n$ thorder time-fractional Boussinesq equation, by constructing iterative processes, we can get the analytical solution successfully. This method is effective and direct, which can be applied to other kinds of nonlinear evolution equations.

\section{Conflict of Interests}

The authors declare that there is no conflict of interests regarding the publication of this paper.

\section{Acknowledgments}

The authors would like to thank the referees and editors for their valuable suggestions and comments which led to a significant improvement of the paper. This work is supported in part by NSFC Grants 11171056, 11271062, and 11401089 and NSIETP 201510200028.

\section{References}

[1] G. W. Leibnitz, "Letter from Hanover, Germany to G.F.A. L'Hospital," in Mathematische Schriften 1849, vol. 2, pp. 301-302, Olms, Hildesheim, Germany, 1695, reprinted 1962.

[2] A. Loverro, "Fractional calculus: history, definitions and applications for engineer," Rapport Technique, Department of Aerospace and Mechanical Engineering, Univeristy of Notre Dame, Notre Dame, Ind, USA, 2004.

[3] D. Baleanu, J. A. T. Machado, and A. C. Luo, Fractional Dynamics and Control, Springer Science \& Business Media, 2011.

[4] V. E. Tarasov, "Fractional generalization of gradient and Hamiltonian systems," Journal of Physics A, vol. 38, no. 26, pp. 5929$5943,2005$.

[5] V. V. Uchaikin, "Self-similar anomalous diffusion and Levystable laws," Physics-Uspekhi, vol. 46, no. 8, pp. 821-849, 2003.

[6] V. E. Tarasov, "Gravitational field of fractal distribution of particles," Celestial Mechanics and Dynamical Astronomy, vol. 94, no. 1, pp. 1-15, 2006.

[7] I. Petras, Fractional-Order Nonlinear Systems: Modeling, Analysis and Simulation, Springer Science \& Business Media, 2011.

[8] S. A. El-Wakil, E. M. Abulwafa, E. K. El-Shewy, and A. A. Mahmoud, "Time-fractional KdV equation for plasma of two different temperature electrons and stationary ion," Physics of Plasmas, vol. 18, no. 9, Article ID 092116, 1994.

[9] S. A. El-Wakil, E. M. Abulwafa, E. K. El-Shewy, and A. A. Mahmoud, "Time-fractional study of electron acoustic solitary waves in plasma of cold electron and two isothermal ions," Journal of Plasma Physics, vol. 78, no. 6, pp. 641-649, 2012.

[10] V. E. Tarasov, Fractional Dynamics: Applications of Fractional Calculus to Dynamics of Particles, Fields and Media, Springer, 2011.

[11] K. Joseph, Fractional Dynamics: Recent Advances, World Scientific, 2012.

[12] N. Laskin and G. Zaslavsky, "Nonlinear fractional dynamics on a lattice with long range interactions," Physica A: Statistical Mechanics and Its Applications, vol. 368, no. 1, pp. 38-54, 2006.

[13] J. L. Bona, M. Chen, and J.-C. Saut, "Boussinesq equations and other systems for small-amplitude long waves in nonlinear dispersive media. I: derivation and linear theory," Journal of Nonlinear Science, vol. 12, no. 4, pp. 283-318, 2002.

[14] J. L. Bona, M. Chen, and J.-C. Saut, "Boussinesq equations and other systems for small-amplitude long waves in nonlinear dispersive media: II. The nonlinear theory," Nonlinearity, vol. 17, no. 3, pp. 925-952, 2004. 
[15] B. Mehdinejadiani, H. Jafari, and D. Baleanu, "Derivation of a fractional Boussinesq equation for modelling unconfined groundwater," European Physical Journal: Special Topics, vol. 222, no. 8, pp. 1805-1812, 2013.

[16] D. Baleanu, K. Diethelm, E. Scalas, and J. J. Trujillo, Fractional Calculus: Models and Numerical Methods, vol. 3, World Scientific, 2012.

[17] A. A. A. Kilbas, H. M. Srivastava, and J. J. Trujillo, Theory and Applications of Fractional Differential Equations, vol. 204, Elsevier Science, 2006.

[18] S. A. El-Wakil and E. M. Abulwafa, "Formulation and solution of space-time fractional Boussinesq equation," Nonlinear Dynamics, vol. 80, no. 1, pp. 167-175, 2015.

[19] P. Zhuang, F. Liu, I. Turner, and Y. T. Gu, "Finite volume and finite element methods for solving a one-dimensional space-fractional Boussinesq equation," Applied Mathematical Modelling, vol. 38, no. 15-16, pp. 3860-3870, 2014.

[20] C. D. Dhaigude and V. R. Nikam, "Solution of fractional partial differential equations using iterative method," Fractional Calculus and Applied Analysis, vol. 15, no. 4, pp. 684-699, 2012.

[21] V. Daftardar-Gejji and H. Jafari, "An iterative method for solving nonlinear functional equations," Journal of Mathematical Analysis and Applications, vol. 316, no. 2, pp. 753-763, 2006.

[22] T. A. Abassy, M. A. El-Tawil, and H. El-Zoheiry, "Modified variational iteration method for Boussinesq equation," Computers and Mathematics with Applications, vol. 54, no. 7-8, pp. 955-965, 2007. 


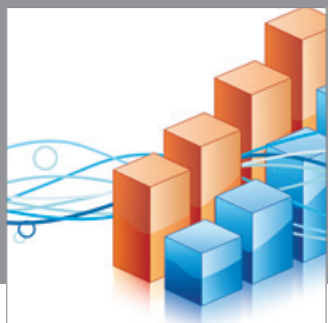

Advances in

Operations Research

mansans

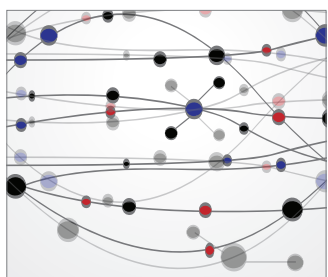

The Scientific World Journal
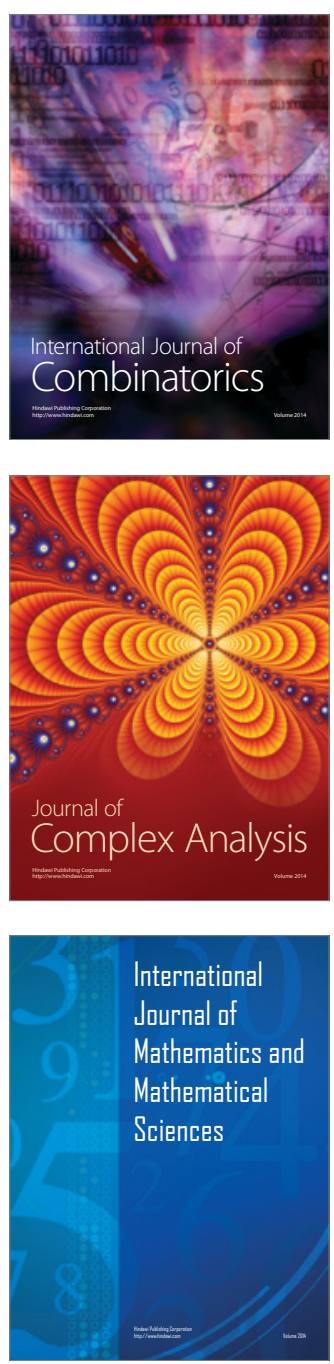
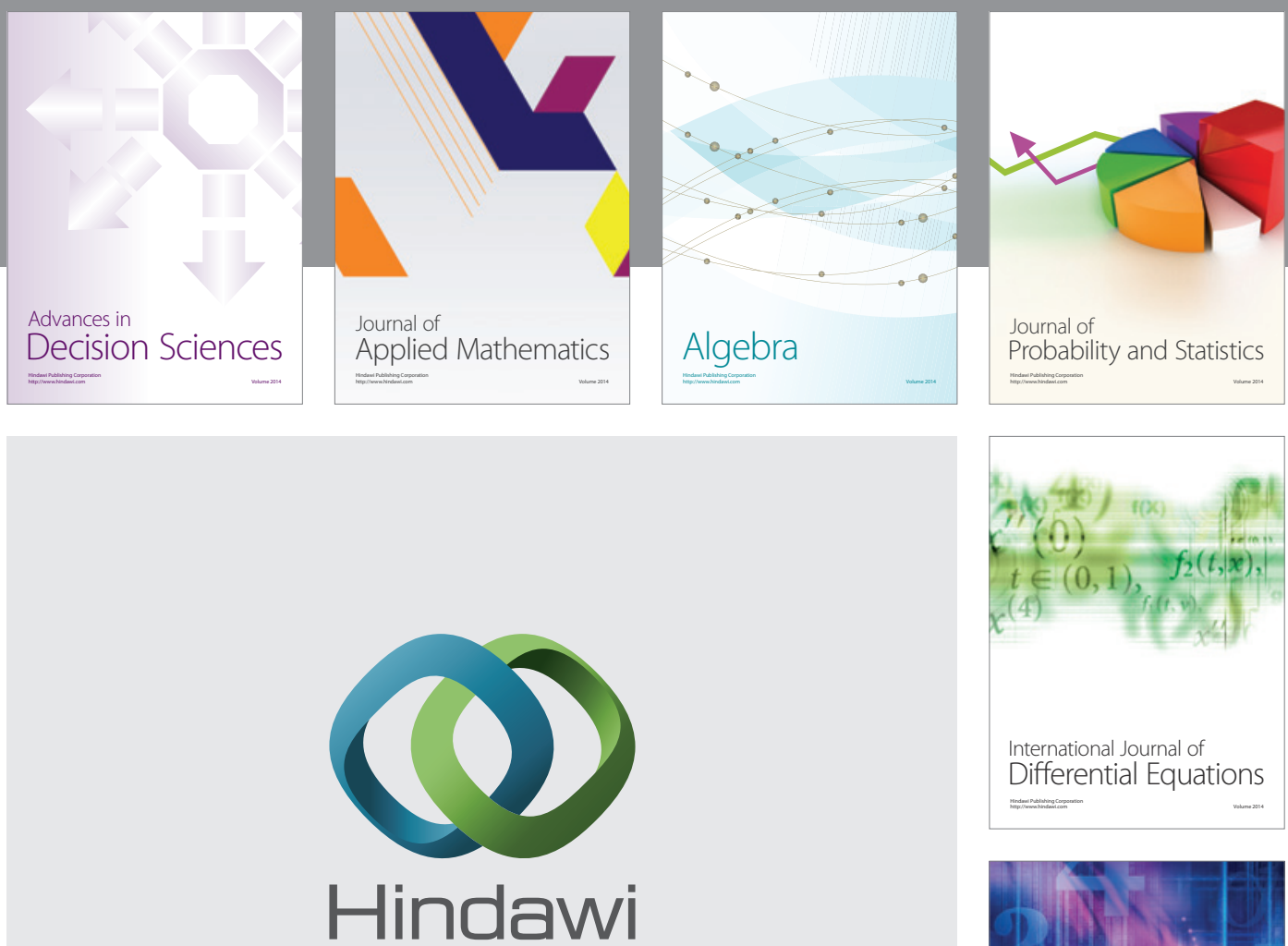

Submit your manuscripts at http://www.hindawi.com
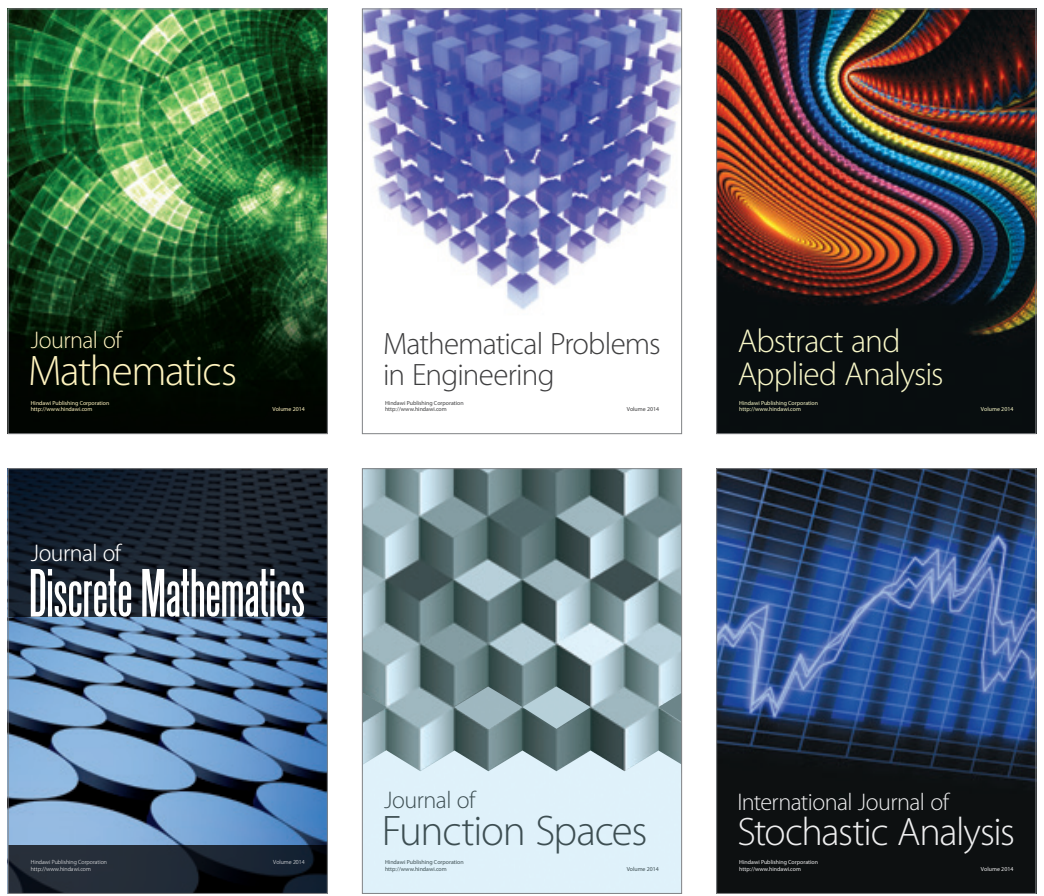

Journal of

Function Spaces

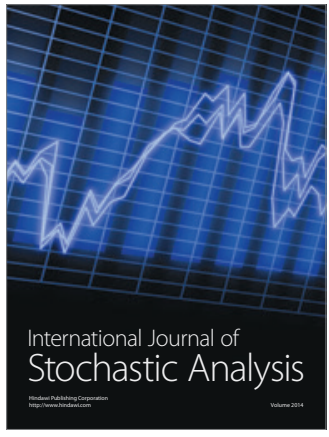

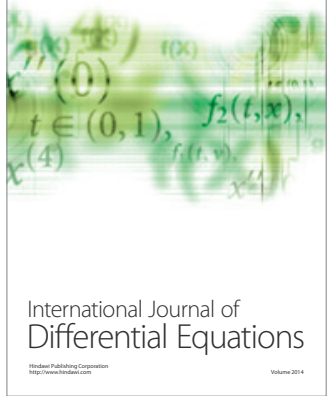
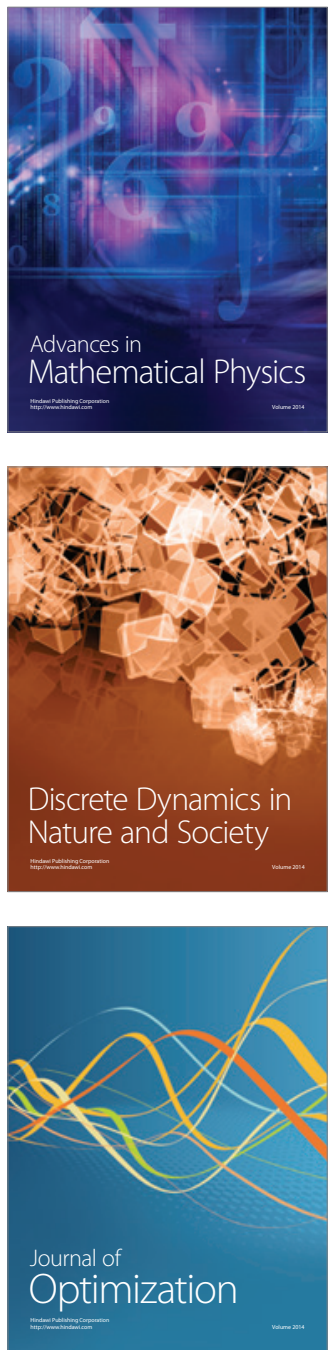